\title{
Versuche über Lichtwirkung bei Hydroa aestivalis (Bazin), Sommereruption (Hutchinson).
}

\author{
Von \\ Prof. Dr. S. Ehrmann, \\ k. k. Primararzt, Vorstand der dermatol. Abteilung des k. k. Krankenhauses Wieden, \\ Wien.
}

(Hiezu Taf. VI.)

Im Jahre 1862 beschrieb Bazin unter dem Namen Hydroa vacciniforme oder aestivalis eine eigentümliche Erkrankung, die er an eine eigens von ihm aufgestellte Form, nämlich an die Hydroa resiculeuse angliederte.

Nach der Beschreibung dürfte die letztere mit den vesikulösen und bullösen Formen des Erythema exsudativum multiforme identisch sein.

Die Hydroa vacciniforme oder aestivalis, von der er selbst sagt: "L'hydroa vacciniforme n'est pas connue des auteurs; l'année dernière j'eus l'occasion d'observer cette singulière éruption" ist hingegen eine Erkrankung sui generis, die nur durch das äußere Aussehen mit der ersteren in Beziehung gebracht werden konnte.

Wie Bazin mitteilt, trat die Erkrankung nach einer Promenade in freier Luft oder nach Einwirkung starker Sonnenhitze auf; zuweilen beginnt die Erscheinung mit Übelbefinden und Appetitlosigkeit. Die Eruption soll zuerst auf den bloßliegenden Körperstellen, dann auch an anderen, besonders auf der Mundschleimhaut auftreten, u. zw. zuerst in Form kleiner, roter Flecken, auf welchen bald durchsichtige Bläschen ent- 
stehen, die denen des Herpes ähneln. Am nächsten Tage sinken sie in der Mitte ein (présentent une ombilication très évidente). Es bildet sich zuerst in der Mitte, dann an der Peripherie des Bläschens eine Kruste und wenn diese sich ablöst, bleibt eine ziemlich starke deprimierte Narbe zurück.

Die Beschreibung, wie sie Bazin gibt, stimmt nur zum Teil mit der späterer Autoren überein.

Viel charakteristischer ist die Beschreibung ron $\mathrm{Hu}$ t$\mathrm{ch}$ inson, namentlich aber seine Abbildungen, die er besonders in dem Smaller Atlas of Clinical Illustrations gegeben hat. Von der einen sagt er: Es handelt sich um ein Mädchen von beiläufig 18 Jahren, das durch mehrere Jahre jeden Sommer ins Krankenhaus aufgenommen wurde. Die Eruption erschien immer mit dem ersten Frühjahrswetter und jeden Herbst verließ die Patientin in verhältnismäßigem Wohlbefinden das Hospital. Doch konnte man nicht sagen, daß sie auch im Winter absolut frei gewesen wäre. Die Eruption bestand in der Bildung von Blasen mit Ulzerationen und hinterließ ausgedehnte Narben. Im Anfange war die Erkrankung auf Gesicht und Hände vollständig beschränkt, später breitete sie sich auf die Schultern aus, schritt aber nie über diese Region nach abwärts und stand deutlich unter dem Einfluß des Sonnenlichts. Ihr Ohr war erodiert, sowie bei gewissen Formen von Frostbeulen und bei anderen Sommereruptionen.

Die zweite Patientin war ein Mädchen von 7 Jahren von sehr feiner Haut, die gegen Sonnenwirkung sehr empfindlich war. Die Abbildung, die Hutchins on gibt, zeigt Gesicht, Hände und Vorderarme nach einer einmaligen Einwirkung der Sonne der ersten heißen Tage des Frühsommers mit Blasen, Rötungen und Borken bedeckt. Von Hutchinson rührt die Bezeichnung Sommerprurigo und Sommereruption her.

Von weiteren englischen Arbeiten sei erwähnt:

Eine Arbeit von Mc. Call Anderson, bei welcher die Krankheit zwei Brüder betraf und kompliziert war durch Anwesenheit von. Hämatoporphyrin im Harn. Auch Jamies on sah die Erkrankung bei zwei Geschwistern. Ferner publiziert Bowen einen Fall mit histologischer Untersuchung der Haut. 
Ferner sind zu nennen: Colcot Fox im Jahre 1895, 1897 und 1899. Dann Radcliffe Crocker im Jahre 1893 und 1900, Graham 1897. Von deutschen Autoren war der erste Berliner, der eine ausführliche Arbeit über Sommerprurigo veröffentlichte im Jahre 1900, Buri 1891, Mibelli 1897.

In allen diesen Fällen wird das Sonnenlicht mehr oder weniger als ätiologisches Moment in Betracht gezogen. Doch wird der Einwirkung anderer physikalischer Agenzien, namentlich höherer oder niedriger Temperaturgrade und dem Einflusse der Luft etc. auch noch eine Wirkung beigelegt.

Physikalische Versuche über Lichtwirkung bei Hydroa vacciniforme sind, soweit ich die Literatur übersehe, nicht angestellt worden. Es war mir deshalb sehr erwünscht, daß ich bei einem Falle, den ich einige Jahre hintereinander zu beobachten in der Lage war, im Herbst und im Winter 1902/03 Versuche über Lichteinwirkung machen konnte. Ich verdanke dies der Liebenswürdigkeit des Herrn Prof. Ed. Lang, der mir gestattete, mit dem damals auf seiner Abteilung befindlichen Finseninstrumentarium die Versuche vorzunehmen, über die ich weiter unten berichte, und wofür ich ihm hiermit, sowie seinen Abteilungsärzten für ihre Unterstützung besten Dank sage. folgender:

Der Fall, an dem meine Versuche gemacht wurden, ist

Schranz Samuel, 31 Jahre alt, Maurer. Pat. ist ein mittelgroßer, mäßig kräftiger Mensch und gibt an, im Alter von 6 Jahren Scharlach gehabt zu haben. Das jetzige Leiden begann im Kindesalter, gleichzeitig an beiden Händen und an den Fingerspitzen, soll sich allmählich über Hohlhand und Handrücken ausgebreitet haben. Auch das Gesicht ist, seit sich Patient zurückerinnern kann, erkrankt. Übrigens ist seit einigen Jahren eine wesentliche Abschwächung des Leidens zu beobachten. Dasselbe Leiden hat nach Angabe des Patienten noch ein Bruder gehabt, der im 32. Lebensjahre nach 8tägiger Krankbeit angeblich an einer Halsentzündung gestorben ist. Auch eine Schwester des Kranken, die gegenwärtig 27 Jahre alt ist, soll daran gelitten haben, befindet sich aber jetzt besser. Von den Eltern ist der Vater im 50. Lebensjahre an Lungentuberkulose gestorben, die Mutter, jetzt 75 Jahre alt, ist angeblich gesund.

Der gegenwärtige Zustand (15. Okt. 1897) bietet folgende Erscheinungen: Die Hände zeigen einen auffallend grazilen Bau und eine Verbildung der Endphalangen, indem die Haut derselben glatt, narbig erscheint und 
straff über die Streckflächen derselben gespannt ist; nur bei horizontalem Druck können einige Runzeln hervorgebracht werden. Das Ende der Phalanx sieht wie abgenützt aus, die Nagelränder wie abgerieben, rissig, verdünnt, der ganze Nagel über das Fingerende bis zur volaren Fläche nach abwärts gebogen, die Phalanx schwer auszustrecken. Die Haut über sämtlichen übrigen Phalangen, über den Gelenken, sowie anf dem Dorsum der Hand bis über das Handgelenk ist narbig, pigmentlos, rosig gefärbt. Auch in den übrigen Gelenken ist die Hand leicht gebeugt und schwer oder nur unvollständig auszustrecken. Die Haut der Flachhand ist auffallend weiß, leicht rosig gefärbt, prall gespannt, verdickt. Diese Beschaffenheit geht bis ins Nagelbett hinein fort. Eigentliche Finger. beeren bestehen kaum. Die Fingerenden sind, wie oben erwähnt, konisch zugespitzt. Auf dem Dorsum der Hand und über den Phalangealgelenken treten zeitweise Blasen auf, die platzen und einer dunklen Borke Platz machen, welche etwa acht Tage festhaftet, sich dann abstöBt und entweder eine granulierende Fläche oder eine rosig gefärbte Narbe hinterläßt. Diesen Vorgang haben wir wiederholt selbst beobachtet. Über dem Handgelenk, unmittelbar über dem Ärmelrand, ist eine etwa 2 Finger breite, leicht pigmentierte Zone, die in die ziemlich weiße und normal aussehende Haut des Vorderarmes übergeht.

Die Nasenwurzel zeigt ebenfalls weiße, rosige, stellenweise narbige Haut, die sich bis nahe ans Jochbein erstreckt. Die Gesichtsknochen scheinen übrigens wenig entwickelt zu sein, besonders der Ober- und Unterkiefer. Die Nasenspitze und die Nasenflügel sind rosig, wie atrophisch oder abgenützt aussehend. Die Ohren, besonders das linke, sind vielfach verkrümmt, zeigen ebenfalls rosige, narbige Stellen und frische Blasen sowie trockene Krusten. Die Haut des Nackens, beiläufig von der zirkulären Linie, welche in der Höhe des Schildknorpels gelegen ist, bis in die behaarte Kopfhaut, hell, rosig, narbig aber glatt, in Falten abhebbar, zeigt an verschiedenen Tagen ebenfalls teils Blasen, teils Krusten. Unter dieser Zone zieht sich eine etwa querfingerbreite, nach unten abklingende, leichtbräunlich pigmentierte $\mathrm{Haut}$, die dann in dieziemlich helle Haut der Brust übergeht. Auf der von der Gewandung vollkommen bedeckten Haut ist keine Spur von Veränderung wahrzunehmen.

Der Patient kam immer im Spätsommer oder im Herbst in mein Ambulatorium und zwar zuerst nur zur Ordination, dann im Winter zur dauernden Pflege; es war dies in den Jahren 1897, 98, 99, 1900, 1902 und 1903 und verließ es dann zum Frühling.

Bis zu Beginn des Monates April, manchmal auch bis Ende April waren dann die Hände ganz frei von Blasen, Krusten und Geschwüren. So wie er im Freien zu arbeiten begann, traten im Beginn kleine Knötchen, dann Blasen, Krusten und Geschwüre auf, die sich abstießen und zu den oben beschriebenen, narbigen Veränderungen der Haut führten. In den letzten drei Jahren kam Patient schließlich nur mehr auf 3-4 Wochen und verließ dann geheilt Wien, um im Frühjahr wieder daselbst in Arbeit zu treten. Im Jahre $1902 \mathrm{kam}$ er erst im Oktober und ich konnte an 
$\mathrm{jhm}$ einige Versuche machen, allerdings nicht so viel, als ich gewünscht hätte. Denn der Mann hatte eine mir nicht erklärliche Angst vor den Versuchen, trotzdem sie ihm weder Schmerzen noch sonstige Ungelegenheiten bereiteten. Er verschwand, ehe ich noch einige Schlußversuche mit Quarzlinsen machen konnte.

Im Jahre 1903 erschien er bloß, um sich für einige Exkoriationen, die er auf den Händen und Ohren hatte, die Heilmittel zu holen. Ich konnte ihn nur mit Mühe in der k. k. Gesellschaft der Ärzte vorstellen. Nichtsdestoweniger geben die angestellten Versuche ein ganz klares Bild über die Ätiologie des Leidens, sie sind rollständig eindeutig, wenn auch die Frforschung gewisser Feinheiten des pathologischen Vorganges durch das eigentümliche Verhalten des Patienten verhindert wurde.

Zur Untersuchung wählte ich die Haut des oberen Teiles der Oberarme, welche bei der Arbeit im Freien von den Kleidern ganz bedeckt, weiß und intakt waren. Sie wurden nicht wie bei Behandlung des Lupus oder anderer Dermatosen durch das Andrücken der von Wasser durchströmten Finsenschen Kompressionslinse aus Bergkrystall blutleer gemacht, sondern die von Wasser durchflossene Linse wurde in variabler Entfernung von der Haut gehalten, so zwar, daß sie an das Ende eines geschwärzten Tubus angebracht wurde, der eine Verlängerung der Finsenschen Lampenröhre bildete, von deren Brennpunkt die Hautoberfläche $15-20 \mathrm{~cm}$ entfernt wurde. Um den Versuch weiter zu variieren, wurden Konvexlinsen sowohl wie Konkavliusen benützt, Linsen von verschiedener Brennweite und die Haut bald innerhalb, bald auBerhalb deren Brennweite zum Vereinigungspunkt gebracht und dadurch die Größe und Lichtintensität des Zerstreuungskreises nach Bedarf geändert.(S.Taf.VI.)

Die Versuche waren folgende: Versuch I (3.,X. 1902): Das Hautniveau wird eingestellt innerhalb der Vereinigungswerte der Strahlen durch das Konvexlinsen-Kompressorium. Dieses $10 \mathrm{~cm}$ vom Brennpunkt der Lampe entfernt. Nach 10 Minuten eine kreuzergroße, dem Zerstreungskreis entsprechende, im Zentrum leicht gedellte, ziemlich stark elevierte, weißlichrote Quadel mit diffusem rotem Hofe. (S. Taf. VI.) Die Rötung ist noch am 10. Oktober sehr deutlich und schwindet dann allmählich. 

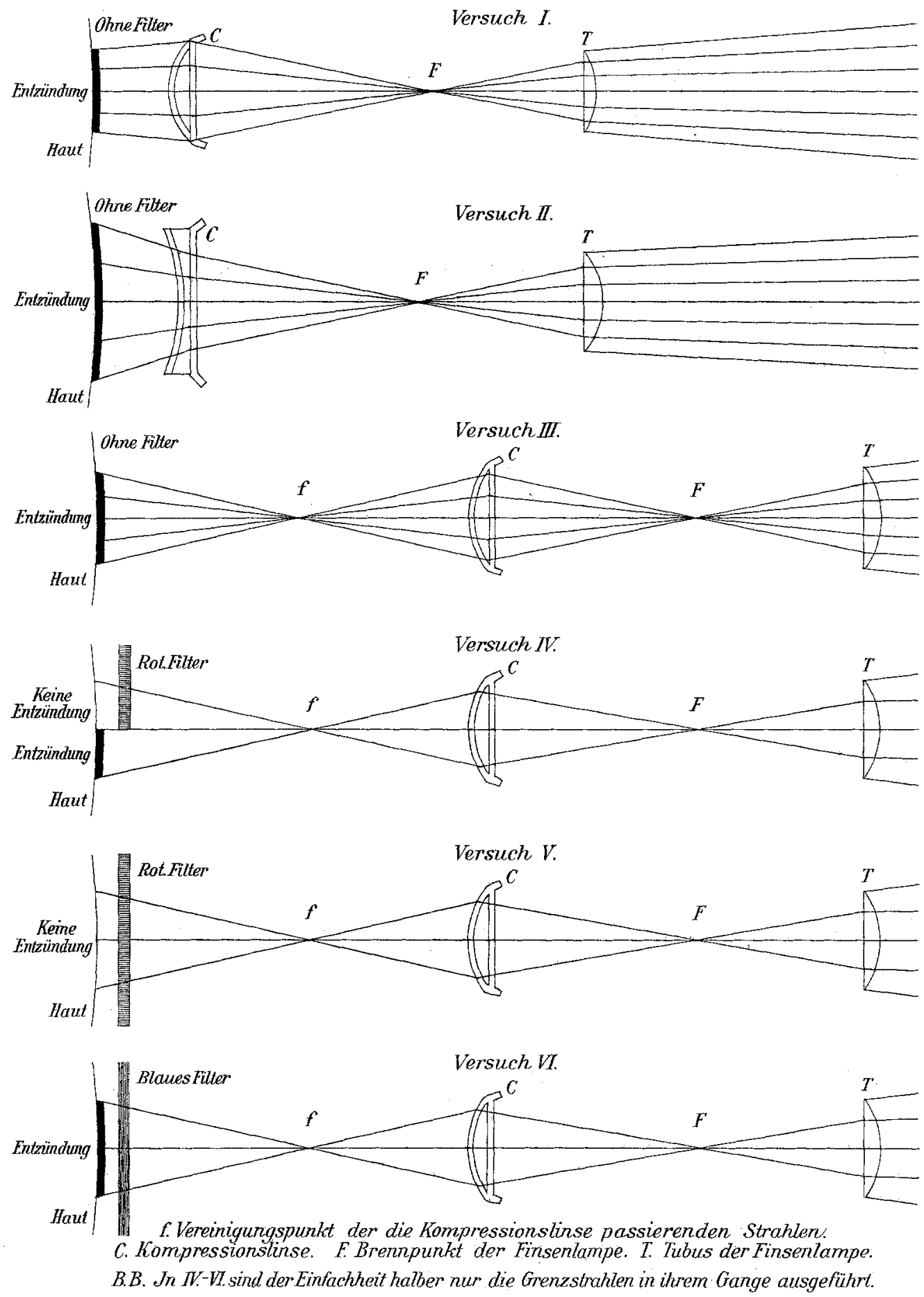

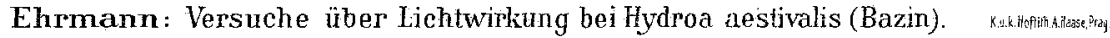


Versuch II (am selben Tage auf dem anderen Arm). Es wird eine konkave Kompressionslinse, $10 \mathrm{~cm}$ rom Brennpunkte der Lampe, benützt. Entfernung der Haut von der Linse $2 \mathrm{~cm}$. Nach einer Viertelstunde schwache Rötung entsprechend dem Zerstreuungskreis, etwa guldengro $B$, diffus begrenzt, in den zentralen Teilen bis aut eine schmale Grenzzone leicht weißlich eleviert.

Versuch III. Konvexlinse. Entfernung $10 \mathrm{~cm}$ vom Brennpunkt der chemisch wirksamen Strahlen der Lampe, $10 \mathrm{~cm}$ außerhalb der Brennweite der Kompressionslinse. Eine Hälfte des Zerstreuungskreises wird gedeckt von einem mit Boraxkarminlösung gefüllten, $1 \mathrm{~cm}$ breiten Glastrog, dessen Seitenwände aus angekitteten Deckgläschen bestehen. (S. Taf. VI.) Bestrahlungsdauer 15 Minuten. An der Grenzlinie zwischen der freiliegenden und der ron Rot gedeckten Hälfte des Zerstreuungskreises entsteht eine linsengroße, in dem unbedeckten Teile liegende bläschenförmige Abhebung der Epidermis, die vom Rot nicht gedeckte Hautpartie zeigt eine hyperämische, halbkreisförmige Fläche. Die von der roten Flüssigkeit gedeckte Hälfte des Kreises ist ganz frei von Entzündungserscheinungen. Die Grenzlinie beider Hälften, die nur von dem Flüssigkeitsmeniscus gedeckt war, zeigt eine blaßrote Farbe. Die Hyperämie der nicht von rot gedeckten Stelle besteht nach 2 Tagen noch fort. Das Bläschen ist zu einer dicken Kruste vertrocknet, die ähnlich aussieht, wie die nach der von der Sonne erzeugten Blase.

Im Herbst 1903 stellt sich Patient vor und wird in der Gesellschaft der Ärzte demonstriert. Es zeigt sich an dieser Stelle eine der Blase entsprechende, deprimierte, umschriebene Narbe, während die übrige Haut ganz normal ist.

Versuch IV (16./X. 1902). Kompressions-Konvexlinse $10 \mathrm{~cm}$ vom Brennpunkt der Finsenlampe. Die Entfernung der Haut $10 \mathrm{~cm}$ vom Brennpunkt der Kompresionslinse. Die Hälfte des Zerstreuungskreises wird von einer roten Rubinglasplatte von $2 \mathrm{~mm}$ Dicke gedeckt. 10 Minuten Beleuchtung. Die gedeckte Hälfte des Zerstreuungskreises vollkommen normal, die nicht gedeckte zeigt eine Quaddel in Form einer halbkreisförmigen Fläche, die sich mit gerader Linie, entsprechend dem Durchmesser des Kreises gegen die rote Partie absetzt. 
Versuch V. Der ganze Zerstreuungskreis wird mit der roten Glasplatte gedeckt. Sonst alles wie im Versuch IV. Die Haut zeigt nach einer Viertelstunde keine Rötung, auch sonstkeine Veränderung (s. Taf. VI). Versuch VI. Experimentum crucis zweimal gemacht. a) Der ganze Zerstreuungskreis gedeckt mit einer $3 \mathrm{~mm}$ dicken Glasplatte von blauem Kobaltglas, $b$ ) mit einem Trog, enthaltend Kupferoxydammoniak, sonst. Versuchsordnung wie in 4 und 5 . (S. Taf. VI.) Nach viertelstündiger Bestrahlung beide Male eine überguldenstückgroße Rötung in Form einer ganzen Kreisfläche, entsprechend dem Zerstreuungskreis mit zentral weißlicher Verfärbung in der Mitte, die sich am anderen Morgen als Epidermisablösung darstellt. An deren Stelle ist nach 2 Tagen eine Kruste, ein Jahr später, 1902, eine deprimierte, linsengroße, scharf umschriebene Narbe zu sehen.

Während aller dieser Versuche war im sehr stark geheizten Zimmer die Zimmertemperatur $20^{\circ}$, die lokale Temperatur an der Hautstelle, wo die Haut von dem Lichtkegel getroffen wurde, innerhalb der Brennweite der konvexen Kompressionslinsen 24:50, außerhalb derselben $235^{\circ}$, bei blauem Lichte $21^{\circ}$, bei rotem Lichte $24^{\circ}$, a. zw. bei Messung mit unberußtem Thermometer.

Wurde zur feineren Messung ein unberußtes Thermometer genommen, um die Reflexion der Wärmestrahlen zu hindern und deren Absorption zu begünstigen, so war die Temperatur innerhalb der Brennweite der Kompressionslinse $33^{\circ}$, außerhalb derselben direkt $30^{\circ}$, bei blauem Lichtfilter $28^{\circ}$, bei rotem wiederum 330. Bei Anwendung der Zerstreuungslinse allein $29.5^{\circ}$ bei berußtem Thermometer, $23^{\circ}$ bei unberußtem.

Ausschlaggebend sind jene Versuche, welche zeigen, daß es mittels der, durch ein blaues Glas filtrierten Strahlen, die dadurch noch wesentlich abgekühlt werden ( 1 Grad über Zimmertemperatur), gelingt, denselben Effekt zu erzielen, wie mittels des direkten Sonnenlichtes, daß es dagegen durch die Strahlen, welche ein rotes Medium passieren, sei es nun eine Glasplatte, sei es eine Flüssigkeitsschichte, obzwar viel weniger oder keine Wärmestrahlen absorbiert werden und die Temperatur viel höher ist, nicht gelingt, auch nur irgend einen Effekt 
zu erzielen. Durch das blaue Medium werden die langwelligen Strahlen des Spektrums und auch der größte Teil der dazu gehörigen Wärmestrahlen absorbiert, es passieren nur die kurzwelligen mit den chemisch wirksamen Strahlen, welche allein die Erscheinungen auf der Haut produzieren.

Beim Filtrieren durch rotes Medium werden von dem Lichte wesentlich nur die kurzwelligen und chemisch wirksamen Strahlen absorbiert, die langwelligen (roten) und Wärmestrahlen werden durchgelassen, sie sind aber unwirksam.

Wir sind deshalb vollkommen berechtigt auszusagen, daß die Erscheinungen, welche bei der Hydroa vaceiniforme oder der Sommereruption beobachtet werden, ein Produkt nur der kurzwelligen und chemisch wirksamen Strahlen des Lichtes sind.

Von besonderem Interesse ist hierbei die in der Krankengeschichte erwähnte pigmentierte Zone am Halse, unmittelbar anschließend an den Rand der Bekleidung sowie auch über den Handgelenken. Ich habe bereits wiederholt in Publikationen darauf hingewiesen, daß die Belichtung durchaus nicht bei jedem Menschen zu gleich intensiver Pigmentierung führt, sondern von der Anwesenheit der Melanoblasten abhängt. Wir wissen, daß albinotische Stellen ned Individuen durch kein Mittel chemischer noch physikalischer Natur zur Pigmentbildung gebracht werden können. Ich habe es auch für die X-Strahlen bewiesen und darauf aufmerksam gemacht, daß schwarzhaarige und brünette Menschen viel intensivere und länger andauernde Pigmentierungen sowohl nach Einwirkung chemischer Agenzien (Vesicatoirs) als nach Lichtwirkung davontragen, daß bei Individuen mit weißer feiner Haut die Pigmentierungen kurze Zeit dauern oder ausbleiben oder nur an solchen umschriebenen Stellen andauern, die schon früher Pigmentzellen (Melanoblasten) trugen wie die Epheliden und Naevi. - Hier in unserem Falle war die sonst bei Arbeitern, die in der Sonne arbeiten, gewöhnlich eintretende Pigmentierung der Gesichtshaut und der Haut der Hände nicht möglich, weil die Lichtwirkung frühzeitig zur Abhebung der Oberhaut und Zerstörung des Papillarkörpers unter Bildung von 
Geschwüren und Narben geführt hat, in welchen keine Pigment bildenden Zellen sich mehr befinden. Dagegen war an den Stellen, die unmittelbar unter dem Rande der Bekleidung sich befanden und rom Sonnenlichte nicht direkt getroffen wurden, das Licht nicht intensiv genug, um die Zerstörungen zu bewirken; es war wegen der Verschiebung des Gewandes immer nur auf kurze Intervalle zur Wirksamkeit gelangt und durch Beugung der Strahlen so abgeschwächt, daß es seine deletäre Wirkung hier nicht entfalten konnte aber trotzdem intensiv genug war, um auf die Pigmentzellen als Reiz zu wirken.

Durch die hier mitgeteilten Versuche werden auch manche widersprechende Angaben der Autoren erklärt. Während nämlich die einen angeben, die Eruption beginne mit kleinen Knötchen, haben andere nur Blasen und Bläschen mit nachfolgender Nekrose gesehen und wiederum andere nur Rötungen allein. Experimentell können alle drei Formen erzeugt werden je nach der Intensität der Bestrahlung.

Da nach Finsens Angaben und den Erfahrungen, die wir täglich bei Bestrahlung normaler oder lupöser Haut machen, eine Entzündung und Bläschenbildung erst 16-24 Stunden nach einer Einzelnbestrahlung (von $1-11 / 4$ Stunden) auftreten, wäbrend bei unserem Falle schon nach einer Bestrahlung von 10-15 Minuten Rötung mit Bläschenbildung vollendet war, der Nekrose nachfolgte, so ist es zweifellos erwiesen, daß wir es hier mit einer abnorm gesteigerten Empfindlichkeitgegen die Wirkung der chemisch wirksamen Strahlen zu t u $\mathrm{n}$ haben. Dies umsomehr, als Finsen Nekrose mit Narbenbildung nie oder vielmehr nur ganz ausnahmsweise bei seiner Behandlung beobachtete und ich eine Kompression der Haut nicht vornabm, diese vielmehr von dem die Lichtwirkung noch mehr schwächenden Blute durchflossen war.

Aus allen diesen Gründen können wir sagen: Di e Sommereruption von Hutchinson oder die Hydroa vacciniformis aestivalis ron Bazin stellt eine familiäre (vielleicht erbliche) Idiosynkrasie der Haut gegenüber der Wirkung der aktinischen Lichtstrahlen dar und ist in Analogie zu bringen 
mit der ebenfalls familiären Epidermolysis bullosa congenita hereditaria, welche eine Überempfindlichkeit gegen mechanische Reize darstelt, nur mit dem Unterschiede, daß bei der letzteren eine Herabsetzung der Idiosynkrasie im Alter, also eine Angewöhnung nicht stattfindet wie bei der Sommereruption. Es könnte also mit Vorteil der Name Sommereruption oder Hydroa vacciniforme, welche über die Ätiologie der Krankheit nichts aussagen, zweckmäßig durch eine andere ersetzt werden, etwa: Epidermolysis oder Dermatolysis photactinicacongenita.

\section{Literatur.}

Bazin. Legons theoriques et cliniques sur les affections géneriques de la peau. Paris 1862.

Buri. Ein Fall von Hutchinsonscher Sommereruption. Monatsh. f. Derm. 1891. Bd. XIII.

Hutchinson. A case of Sommer-Eruptions recurring with great severity for many years etc. Clinical society transactions. 1888.

Derselbe. Sommer-Eruptions. Verhandl. des II. Dermat.-Kongr. Wien 1892.

Jamies on. Lancet 1888.

Berliner. Über Hutchinsons Sommerprurigo etc. Monatshefte f. Dermat. 1890.

Crocker Radcliffe. Hydroa vacciniforme. 1893.

Bowen. Hydroa vacciniformis, Bazin, Hutchinsons Sommereruption with histological examination. Journ. of cutan. and gen. urinary diseases. 1894. Bd. XVII.

Brocq. De l'hydroa vacciniforme. Ann. de derm. et de syph. 1894. Jaris ch. Demonstr. Verhandl. d. V. Kongr. d. d. derm. Ges. 1895. $\mathrm{Hutchinson.} \mathrm{A} \mathrm{smaller} \mathrm{Atlas} \mathrm{of} \mathrm{clinical} \mathrm{surgery.} 1995$.

Graham. Hydroa aestivale. Journ. of cut. and gen.-ur. dis. 1897. Mibelli. Die Histologie der Hydroa vacciniforme von Bazin. Monatshefte f. Derm. 1897. 